\title{
Intelligent Energy Management System for Residential and Community Applications
}

\author{
Vicente Botón-Fernández ${ }^{1}$, Máximo Pérez Romero², \\ Adolfo Lozano-Tello $^{1}$, and Enrique Romero-Cadaval ${ }^{2}$ \\ ${ }^{1}$ Quercus Software Engineering Group \\ University of Extremadura, Escuela Politécnica, Campus Universitario, s/n \\ 10071 Cáceres, Spain \\ ${ }^{2}$ Power Electrical and Electronics R+D group \\ University of Extremadura, Avda. De Elvas, s/n \\ 06006 Badajoz, Spain \\ \{vboton, mperez, alozano, eromero\}@unex.es
}

\begin{abstract}
This paper presents a Smart Storage System able to manage the energy and the smart home devices of a house for optimizing the local consumption of energy, even if there is not renewable generation. The proposed system is composed by two main systems. On the one hand, the Local Energy Management units that will be located in the houses, which are able to maintain the power consumption under a maximum reference value and to switch on/off the devices in the house by using domotic protocols. On the other hand, the Central Energy Management and Intelligent System, that receives operation data from each local unit, analyzes them using behavioral and optimization algorithms and determines the best way in which each local unit has to operate, communicating the operation references back to them.
\end{abstract}

Keywords: Smart Energy Storage, Intelligent Systems, Pattern Recognition, Ontologies, SWRL.

\section{Introduction}

The availability of having energy storage systems (ESS) [1, 2] is a key factor for establishing new operation strategies in electric distribution grids related with the hot topic of Smart Grids. This ESS will do easy to integrate distributed generation systems based in renewable energies placed in houses, residential and commercial building and also will contribute to have some control on the load profile, and so achieving a better overall performance and utilization of the distribution grid.

Although this kind of systems has been widely used associated to distributed generation plants, mainly with photovoltaic installations [3, 4], they could be used without any energy generation systems with the aim of smoothing the load curve or even control the consumption of energy depending on its price-by-hour [5].

An important feature that these environments need to possess is the ability to adapt themselves to the residents' consumption habits and have the versatility to make 
decisions in a variety of situations. In this sense, finding consumption patterns in a sequence of events in order to predict future peaks on the load curve, can lead us to the energy optimization we are looking for. Therefore, the system will be able to recognize power consumption patterns and determine the best way to smooth future peaks. Obviously, discovering these patterns requires a previous task of learning. In a smart grid environment, learning means that the system has to gain knowledge about the use of energy and common behavior of the electric distribution grids in an unobtrusive and transparent way.

Using ontologies [6] to classify the types of appliances and sensors and their functionality can be an appropriate way to understand the load profile of a house or residential building. Ontologies and SWRL (Semantic Web Rule Language) rules provide a precise definition of smart grid taxonomy and are reusable, so other systems can get them to classify their own components and to build rules that will allow inferring new information.

In this paper an Intelligent Energy Management System (INTELEM) is presented. This system is composed by Local Energy Management Units (LEMU) and a central system that receives data from a group of LEMUs by conventional connections. This central system analyses that information using behavioral algorithms in order to decide their actions and also to be communicated with the distribution grid operator, so that it can manage efficiently the energy. In this way, the system becomes a Central Energy Management and Intelligent System (CEMIS).

\section{Contribution to Internet of Things}

This work merges two areas: energy storage and Information and Communications Technology (ICT), providing an autonomous power consumption data capture and event transfer through TCP/IP. A central server analyzes the information transmitted over the network using smart algorithms to optimize the load curves in residential or community environments.

\section{Existent Work about Intelligent Energy Management Systems}

Nowadays, the development of data mining techniques to recognize habitual behavior and provide feedback in context constitutes a key factor to empower individuals to take control over residential electricity consumption. There are several factors that drive household electricity consumption behavior, such as energy-related attitudes, socio-demographic factors, building characteristics, energy prices, etc. van Raaij and Verhallen [7] recognize this problem and suggest that habits can become alternative predictors of electricity consumption, because routines or habits may resist the cognitive and financial drive and still prevail over rational alternatives.

In this sense, Joana M. Abreu et al. [8] propose a methodology which demonstrates that it is possible to use pattern recognition methodologies to identify habitual electricity consumption behavior given the intrinsic characteristics of the residents. 
The present work aims to achieve similar objectives but with a different approach. It presents an intelligent system that recognizes electricity consumption patterns to automate the grid behavior in an efficient way, providing electricity supply when needed and saving as much energy as possible. The algorithms are based on the analysis of temporal energy consumption data and the use of the building resources. In spite of the limit literature about ontologies and production rules in smart grid systems, this work uses both of them to automate the behavior of the building.

\section{INTELEM}

The Intelligent Energy Management System presented in this paper, is built upon an existing project called IntelliDomo [6]. It is a smart system able to control the devices of a home automation system automatically and in real time using SWRL rules. Its main feature is the ability of reasoning and responding in view of the continuous changes in domotic environments.

Each event captured from sensors and actuators is recorded. To manage all this knowledge about the environment, this system uses an ontology called Domo OWL, whose concepts have been modeled to be in sync with the physic devices that constitute a home automation environment, so that it can be stored its outstanding values and properties. Furthermore, the ontology works together with a set of established SWRL rules. The users can manage these rules or create new ones through IntelliDomoRules tool, automating the system behavior as well.

There also exists a domotic database (QDS_DB) where IntelliDomo updates the state values of the physic devices in real time. This way, IntelliDomo is in charge of transmitting the changes instantly from physical (devices) to logical (database) levels and vice versa, maintaining the data integrity.

Finally, there is a learning module which incorporates algorithms in order to acquire users' habits and automatically generate production rules for behavior patterns to anticipate the user's periodic actions. As a result, the system can adapt to changes in the discovered patterns based on the user implicit and explicit feedback.

INTELEM uses some of the above modules to achieve its objectives. Its architecture is presented in Fig. 1. As it can be seen, the CEMIS can take control of

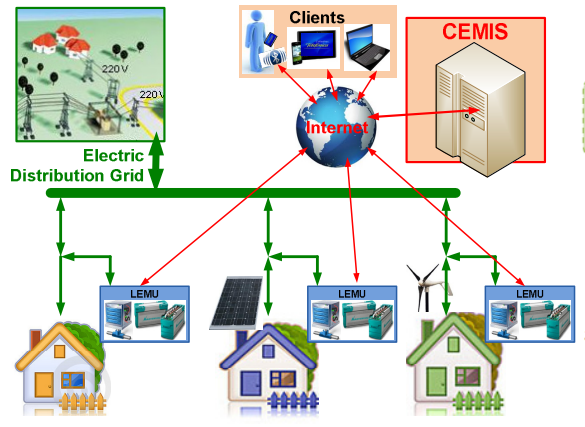

(a)

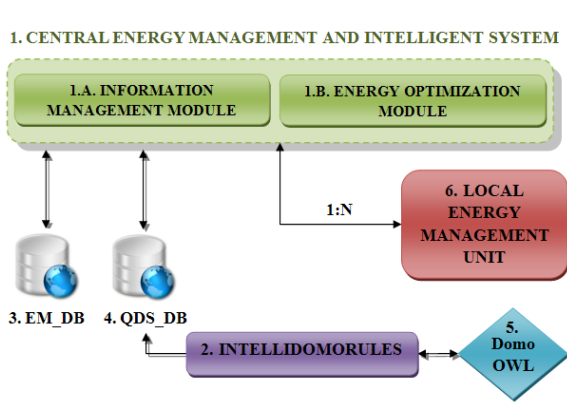

(b)

Fig. 1. Main components of the INTELEM architecture (a) and their relations (b) 
several (n) LEMUs and is composed by two main modules: the Information Management Module (IMM) and the Energy Optimization Module (EOM). It's important to remark that these LEMUs are distributed over different houses or residential buildings whereas the CEMIS is located at a central server, using a conventional connection via TCP/IP to exchange information with all these LEMUs.

On the one hand, the CEMIS receives data (energy consumption, battery state, etc) from a group of LEMUs and stores it into a central database called EM_DB. On the other hand, the CEMIS analyzes periodically the data logs with different behavioral algorithms in order to recognize energy consumption patterns and use this information to generate production rules which can optimize the use of the grid and save energy. These rules are stored into the QDS_DB database and managed through the IntelliDomoRules tool. Finally, once the rules are fired, the corresponding changes are transmitted, via control signals, to the affected LEMUs.

In the following sections we're going to check in depth each of these modules, with special emphasis on the CEMIS structure.

\subsection{Local Energy Management Unit}

This unit is designed to be placed inside houses or residential buildings with the aim of controlling their energy consumption, and it corresponds to the general scheme shown in Fig.2, having the objectives described below.

In the first place the LEMU is able to limit the power consumption peaks, which can take place due to start-up transients or high power devices operating during shorttime intervals, by supplying extra energy from the ESS. As a result, the user could contract with the electric power company to reduce one or two steps the level of contracted power that he would normally have without this device. As this contracted power is usually included as a fixed cost in the electricity invoice, the user could have direct economic benefits.

In the second place the LEMU can decouple the consumed energy from the energy demanded to the grid. As far as time-of-use (TOU) rates are concerned, it would be possible to charge the ESS from the grid during the off-peak hours (cheaper prices)

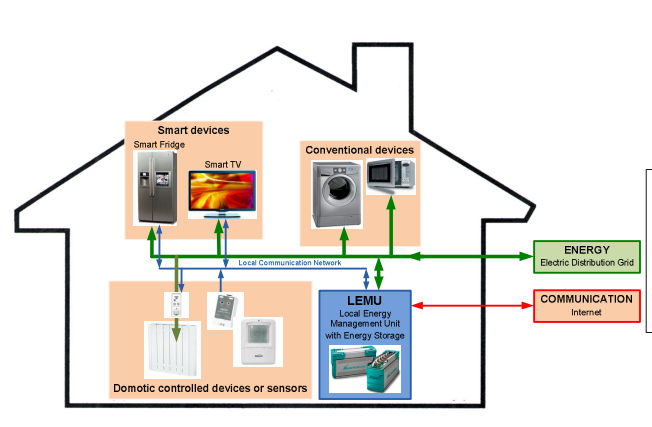

(a)

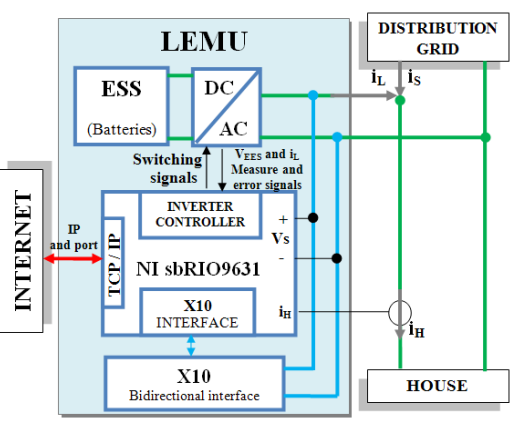

(b)

Fig. 2. Schematic diagram of: (a) the interconnection of home appliances, and (b) the Local Energy Management Unit (with Energy Storage System) 
and then supply its energy to the devices during the peak hours (higher prices). In our ongoing work, we plan to let the LEMU work as a constant power sink, by using the ESS to put the difference of energy between the energy demanded from the grid and current level of energy consumed by the devices into the house or building.

Otherwise, the LEMU can switch on or switch off the devices by using the X10 protocol to maintain the power consumption under an established limit, and it can also receive the X10 events sent by X10 sensors such as motion sensors.

Finally, the LEMU has been designed to be integrated into an energy management network, and it has the possibility of being connected to a central system in order to send local data, such as electrical magnitudes (like smart meters do), the state of charge of its ESS or X10 events. The LEMU can also receive operation references from the central system such as power consumption limits, or some commands to switch on/offX10 actuators.

\subsection{Central Energy Management and Intelligent System}

This central system is in charge of controlling the group of LEMUs which are distributed over the energy management network. It's designed to be hosted in a remote server and its main objective is to efficiently manage the energy consumption of a house or building through data mining algorithms.

Basically, the LEMUs gather power consumption data through the sensors that are connected to the grid, sending the information to the CEMIS. Once the CEMIS receives that information, it can analyze it and then use it to send back control signals to the LEMUs to control their behavior and save energy. As it was mentioned before, the CEMIS has two different modules to achieve those objectives: the IMM and the EOM. Next, we describe the operation of these modules and the relations between them.

\subsubsection{Information Management Module}

This module is continuously reading and storing into a database the information provided by the LEMUs. The Fig. 3 describes the general operation of this module. The data sent by the LEMUs can be values of electrical magnitudes, X10 events or the state of charge of an ESS. The electrical magnitudes are often sent every 5 seconds, whereas the other values are sent when a certain event takes place (e.g. an X10 sensor detects motion).In order to better identify each type of information, it has been defined three different message format, all of them with the following structure: header field plus data field.

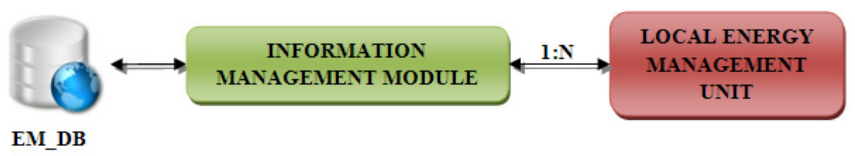

Fig. 3. Information Management Module schema 
The X10 message format is as follows: header = "X10", data $=$ \{house code, unit code, value $\}$. The data field uses one character to represent the house code (A-P), two characters to describe the unit code (1-16) and three more characters to identify the value. The ESS message format has the following fields: header = "BAT", data = state of charge (\%).Finally, the power consumption message format uses the data field to store the value of electrical magnitudes $(\mathrm{kW})$ and its header is "CON".

Otherwise, these messages are transmitted through a communication channel(or socket) between each LEMU and the CEMIS. In this case, it's the LEMU who takes the client role and the one who initiates the communication session with the CEMIS, which is waiting for incoming requests. Once the socket is established, the LEMU will send data packets continuously, and then the IMM will process them and save the information into the EM_DB. This way, the database keeps a log of everything that happens inside the houses or residential buildings. Obviously, after each packet reception the IMM will response with a message indicating if the data was correctly saved into the database or not. If there is any error, the packet will be send once again, but there is a limit number of attempts.

\subsubsection{Energy Optimization Module}

The main objective of this module, which is still in development, is to coordinate a group of LEMUs, optimizing the use of the grid and saving energy. To that end, this module will incorporate data mining algorithms with the purpose of controlling the ESS charging time. These algorithms will aim to discover the resident power consumption patterns and try to have the ESS prepared (full charge) for those moments where the consumption is considerably high (peak periods). With this information, the system could predict future demands on the grid and manage them with the best strategy. Fig. 4 sums up the operation of this module.

First of all, the EOM reads the log entries from the EM_DB database. These entries are composed by three fields: energy consumption value, date and time of the event and IP of the associated LEMU. Then, the EOM selects a specific LEMU and sends the corresponding entries, based on the LEMU's IP, to the data mining algorithms.

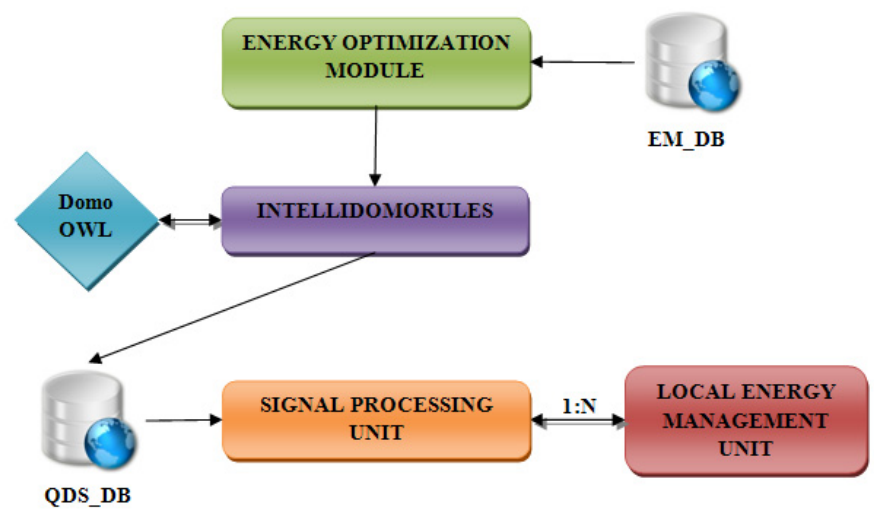

Fig. 4. General view of the Energy Optimization Module 
Then, the algorithms will analyze this data set and look for frequent peaks in the load curves (see Fig. 5). Once they recognize the patterns, they will design a strategy to coordinate the behavior of the LEMU and optimize the use of the resources. The way to carry out this strategy involves generating SWRL rules that automate the corresponding actions. Therefore, the EOM will send the results of the data mining process to the IntelliDomoRules tool. This one can build the rules and save them into the ontology (Domo OWL) and the database (QDS_DB).

The Signal Processing Unit (SPU) monitors the rules of the QDS_DB database. When a rule affecting a LEMU is fired, it sends a control signal to the associated LEMU. There are two types of control signal: one to control the charge of the ESS and another one to manage the X10 devices. The control signals for X10 devices follow the same format as that used in the IMM-LEMU communication, but now these signals can remotely modify the physical state of an X10 device. This type of signal is usually used to switch non-priority X10 devices off when the consumption curve reaches a peak and there's no enough electricity supply in the ESS. Otherwise, the first type of signals has a header value of "BAT" and it uses the data field to store the ESS command, which would be "on" if the EOM wants to start the charge of the ESS or "off" if it wants it to stop the charge. To communicate the SPU with a LEMU it's necessary to create another socket. However, this time it's the CEMIS who takes the role of the client and each LEMU will work as a server, listening for new requests. The SPU sends a control signal and then receives an acknowledgement response from the LEMU, which can be a message like "OK", "ERROR", etc. Once the control signal has been correctly transmitted, the socket is closed and the LEMU waits again for new requests.

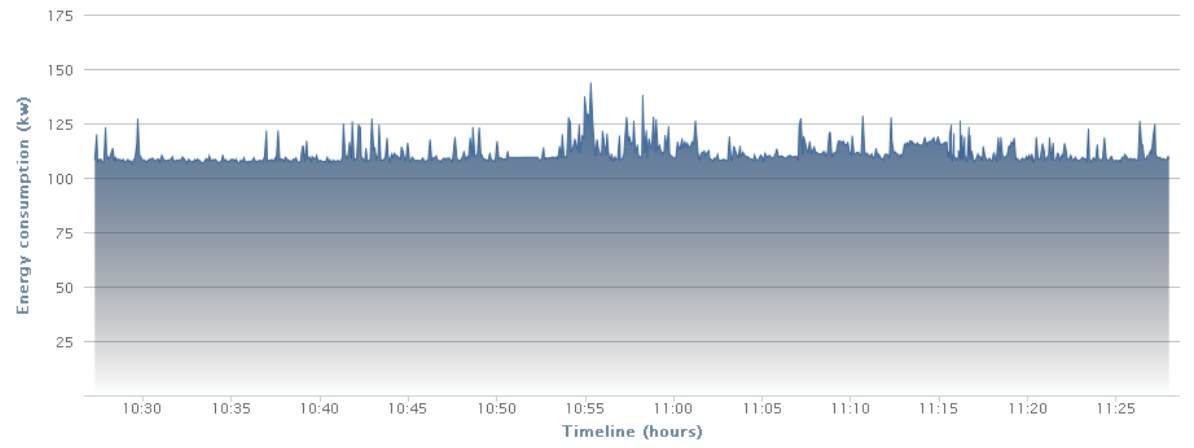

Fig. 5. Example of the power consumed over time in our lab. This data can be checked at anytime at http://158.49.245.68:8810/ecosaver/app/events/events.jsp.

\section{Conclusions and Future Works}

There are many opportunities to use INTELEM (Intelligent Energy Management System) in Smart Houses or Buildings to achieve economic benefits and an optimized use of the distribution grid. These opportunities increase if the house or building has a 
distributed generation system, which is another factor that must be taken into account when defining the operation strategy. In this sense, this paper presents the bases of an intelligent energy management system that aims to achieve these objectives, describing the relations and the main features and functions of each component.

At present, INTELEM can control several LEMUs (Local Energy Management Units) to optimize the power energy consumption and manage smart home devices remotely. In our ongoing work, we plan to design data mining algorithms in order to coordinate the behavior of a set of LEMUs in an efficient way, so that we can optimize the use of the distribution grid in different houses at the same time while saving energy altogether. We will test these behavioral algorithms in different scenarios to better understand their strengths and weaknesses.

Acknowledgements. This work has been developed under support of Telefónica Chair of the University of Extremadura, FEDER and Junta de Extremadura.

\section{References}

1. Pieper, C., Rubel, H.: Revisiting Energy Storage: There Is a Business Case. The Boston Consulting Group (2011)

2. Guerrero, M., Romero, E., Barrero, F., Milanés, M., González, E.: Supercapacitors: Alternative Energy Storage Systems. Przegląd Elektrotechniczny (2009)

3. Smart Energy Storage System, http: //panasonic.net/energy/storage_battery/index.html

4. Towards a "Lifestyle with Virtually Zero CO2 Emissions throughout the Entire House", http: / / panasonic.net/eco/zero-co2/

5. Auväärt, A., Rosin, A., Belonogova, N., Lebedev, D.: NoordPoolSpot Price Pattern Analysis for Households Energy Management. In: 7th International Conference-Workshop Compatibility and Power Electronics, pp. 103-106. Tallinn Univ. of Technol., Tallinn (2011)

6. Botón-Fernández, V., Redondo-García, J.L., Lozano-Tello, A.: Learning Action Sequences for Decision-Making in Home Automation Systems. In: 7th Iberian Conference on Information Systems and Technologies, pp. 126-131. AISTI, Madrid (2012)

7. van Raaij, F., Verhallen, T.: A Behavioral Model of Residential Energy Use. Journal of Economic Psychology 3, 39-63 (1983)

8. Abreu, J.M., Câmara Pereira, F., Ferrão, P.: Using pattern recognition to identify habitual behavior in residential electricity consumption. Energy and Buildings 49, 479-487 (2012) 